http://jmscr.igmpublication.org/home/

ISSN (e)-2347-176x ISSN (p) 2455-0450

crossref DOI: https://dx.doi.org/10.18535/jmscr/v7i7.92

\title{
A Comparative Study of Clinical Profile of Dengue in Patients with and without Diabetes Mellitus Admitted in a Tertiary Care Centre in Rural South Kerala
}

\author{
Authors \\ Dr H Poornima ${ }^{1}$, Dr Arathi $\mathbf{N}^{2}$ \\ ${ }^{1}$ Additional Professor, Department of Medicine, Government TD Medical College, Alappuzha \\ ${ }^{2}$ Assistant Professor, Department of Medicine, Government TD Medical College, Alappuzha \\ Corresponding Author \\ Dr Arathi N \\ Assistant Professor, Department of Medicine, Government TD Medical College, Vandanam PO \\ Alappuzha: 688005, India
}

\begin{abstract}
Background: Dengue is one of the most common infection in the tropical countries. Comorbidities, especially Diabetes is known to influence the outcome of patients with Dengue fever.

Objectives: To study the clinical profile of patients with dengue and to identify the role of Diabetes mellitus in the development of sever dengue.

Methods: Observational study on 342 patients admitted with a diagnosis of Dengue fever. Patients were admitted and managed as per the institutional protocol. The patients were studied during the entire hospital stay and was observed for the development of complications like hemorrhage or shock. The differences in occurrence of complications among diabetics and non diabetics were compared.

Results: Complications like Dengue Hemorrhagic fever, Shock and mortality were high among diabetics when compared to non diabetics.

Conclusions: Diabetic patients admitted with dengue requires more vigilant monitoring for development of complications.
\end{abstract}

\section{Background}

Dengue is an important mosquito borne disease in the tropical and subtropical regions. Dengue fever is often clinically self limited and presents with fever, arthralgia, myalgia and head ache. Dengue hemorrhagic fever is characterized by thrombocytopenia, hemorrhage and plasma leak resulting from increased vascular permeability ${ }^{(1,2)}$. Dengue shock syndrome has a mortality of as high as $20 \%{ }^{(3-5)}$.

Co-morbidities were reported to aggravate dengue illness, suggesting host characteristics risk factors in the development of Dengue Hemorrhagic fever/ Dengue Shock Syndrome ${ }^{(6-9)}$. Diabetes Mellitus. a globally highly prevalent disease, is one of the note worthy proposed host risk factors for the development of Dengue Hemorrhagic Fever/Dengue Shock Syndrome/ Severe Dengue, although conflicting results were found in some researches; this is not surprising as Diabetes is a multifaceted disease that implicates a wide range of metabolic derangements and immune dysfunction. 
The objectives of the study were to identify the role of Diabetes Mellitus as a risk for the development of serious forms of Dengue, emphasizing the role of blood sugar control.

\section{Aims and Objectives}

1. To study the clinical profile of patients admitted with Dengue fever.

2. To understand the role of Diabetes Mellitus as a probable risk factor for the development of severe forms of Dengue.

\section{Materials and Methods}

Study Population: Patients admitted with the diagnosis of Dengue fever from the June December 2018 in a tertiary care centre in rural south kerala.

Study Design: Observational study

\section{Inclusion Criteria}

a. Age more than or equal to 13 years of both the sexes.

b. Patients satisfying the WHO definition for Dengue.

\section{Exclusion Criteria}

a. Those who are not willing to give a consent.

b. Those with known pre existing Thrombocytopenia or coagulation disorders

Methods: Among the patients presenting to the outpatient department or emergency department of the institution who satisfied the inclusion criteria were enrolled in the study.

After history taking and physical examination the following investigations will be performed.

1. Blood Samples for: Complete Blood Counts, Erythrocyte Sedimentation Rate, Blood Urea, Serum Creatinine

2. Random Blood Sugars, Fasting and post prandial blood sugars and HbA1c will be done. Patients will be classified as diabetics or not based on the ADA definitions.

3. Ultrasound abdomen in relevant cases.
The patients will be hospitalized according to the institutional protocol and managed as per the WHO guidelines. Patients will be monitored clinically for the development of Dengue Shock Syndrome/ Dengue Hemorrhagic Fever. Daily CBC will be measured for the evidence of hemoconcentration.

Patients will be followed up till the end of their hospital stay with the outcome measured as either died or survived.

After data collection, an attempt will be made to find out the effect of Diabetes mellitus, either pre existing or newly detected Diabetes, in the development of complications and the final outcome.

\section{Definitions}

WHO 2009 definitions of Dengue fever:

1. Probable Dengue fever:

An acute febrile illness with 2 or more of the following:

- Retro orbital pain

- Myalgia and arthralgia

- Nausea and vomiting a

- Skin rash

- Hemorrhagic manifestations. AND

Supportive serology OR

Occurrence at the same location and time as other confirmed cases of Dengue fever

2. Confirmed case of Dengue fever: Confirmation of Dengue fever is baed on laboratory criteria

Isolation of virus from serum or tissue sample

OR

Demonstartion of 4 fold or more rise in $\mathrm{Ig}$ $\mathrm{G}$ and $\mathrm{Ig} \mathrm{M}$ antibody titres to the dengue antigens in paired serum samples

OR

Demonstration of dengue antigens in tissue, serum, CSF by immunohistochemistry, immunoflourescence or ELISA 
OR

Detection of genomic sequences by PCR

Dengue Hemorrhagic Fever: Requires all 4 of the following to be satisfied:

a. Acute fever lasting 2-7 days occasionally bi phasic.

b. Hemorrhagic tendencies as evidenced by atleast one of the following: a positive tourniquet test, petechiae, ecchymosis purpura, bleeding from mucosa, hematemesis or malena

c. Thrombocytopenia $<100000 / \mathrm{mm} 3$

d. Plasma leakage as evidenced by at least one of the following: Rise in hematocrit > $20 \%$, Fall in hematocrit $>20 \%$ after IV fluids, Pleural effusion, ascites or hypoalbuminemia.
Dengue Shock Syndrome:

The identification of Dengue shock syndrome requires all 4 of the dengue hemorrhagic fever criteria and evidence of circulatory failure as manifested by:

1. Rapid and weak pulse

2. Narrow pulse pressure $(<20 \mathrm{~mm} \mathrm{Hg})$

3. Hypotension for age $(<90 \mathrm{~mm} \mathrm{Hg}$ for age $>$ 5 years)

4. Cold clammy skin, restlessness.

ADA 2018 definitions of diabetes:

Fasting Plasma Glucose of $\geq 126 \mathrm{mg} / \mathrm{dl}$

OR

2 hour Post prandial Plasma Glucose $\geq$ during OGTT

OR

$\mathrm{HbA} 1 \mathrm{c} \geq 6.5 \%$

OR

In a patient with classic symptoms of hyperglycemia or hyperglycemic crisis, a random glucose $\geq 200 \mathrm{mg} / \mathrm{dl}$

\section{Observations}

Figure 1: Age Distribution Of Cases

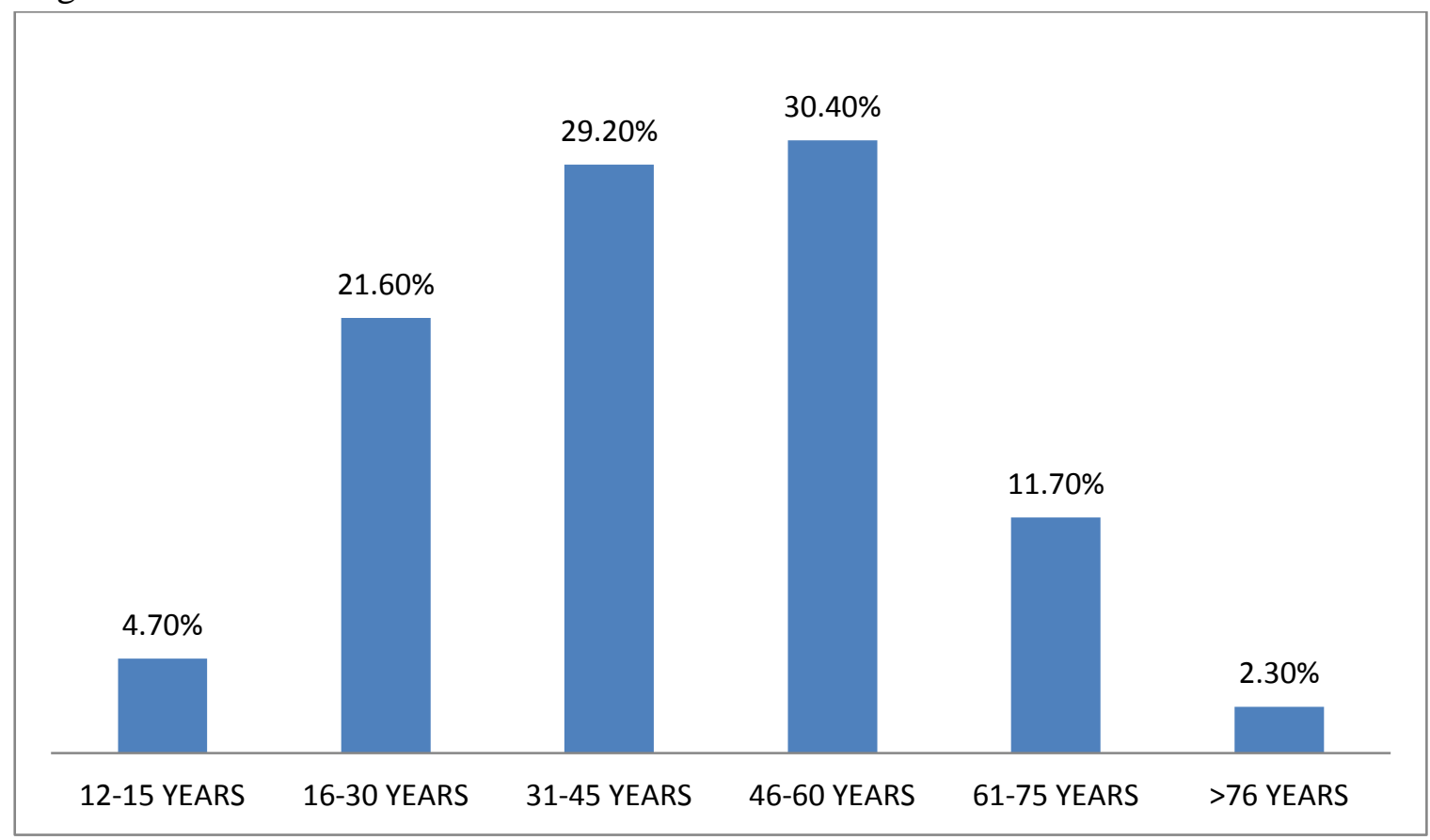

Most of the patients were in the age group of 16-60 years of age, which represents the most productive of the age groups.

$31 \%$ of the cases were females and $69 \%$ were males. 


\section{JMSCR Vol||07||Issue||07||Page 511-518||July}

Figure 2: Symptoms among Patients Admitted with Dengue

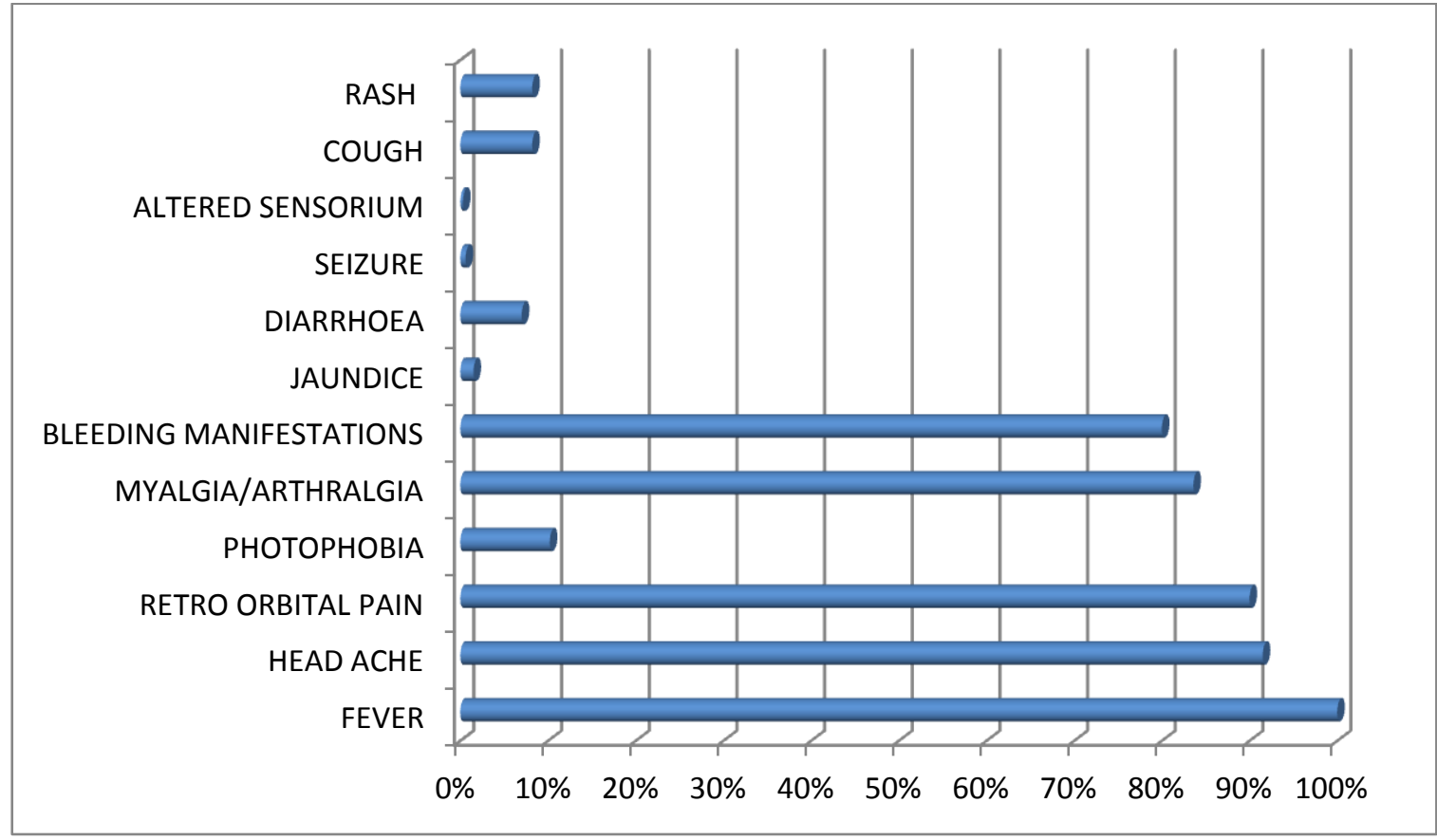

Figure 3: Types of Bleeding Manifestation among the cases

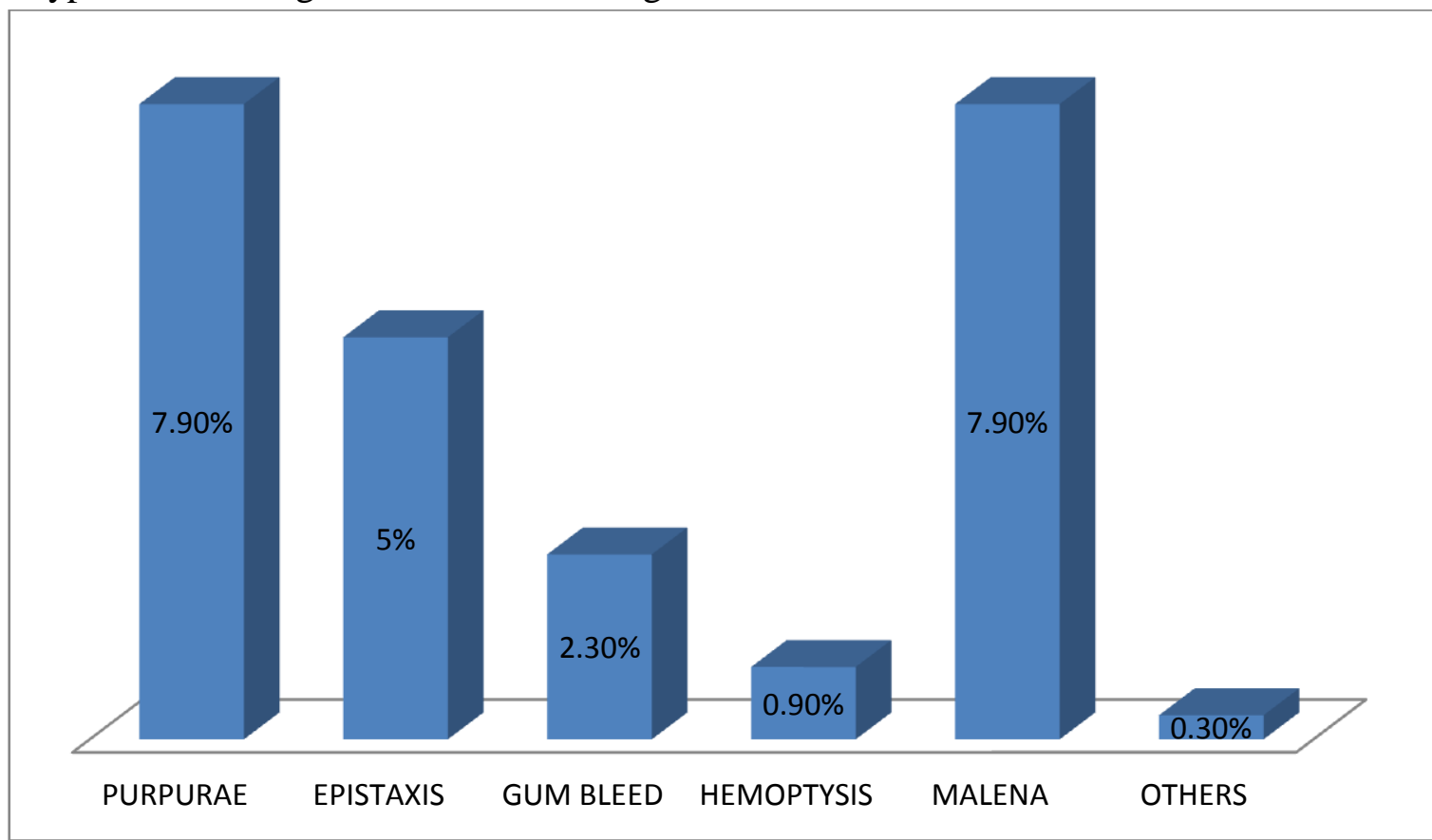

$84.3 \%$ of the cases had fever duration between 2-7 days. $5.3 \%$ cases had fever of $<2$ days duration and $10.5 \%$ cases had fever more than 7 days. Most of the cases had an average stay of 3-6 days in hospital(63.7\%). $19.9 \%$ had hospital stay of 6-9 days duration, $7 \%$ had more than 9 days duration. $5.8 \%$ were discharged in 3 days.

$50 \%$ of the patients had a total count of $<4000 /$ cubic millimeter during admission. 5.6\% had a total count of $>10,000$. $51.5 \%$ of patients had platelet count of $<10,000 /$ cubic millimeter. None of our patients had a platelet count of $>1$ lakh at the time of admission, probably because ours was a tertiary care centre. Platelet count recovered to $>70,000$ /cubic millimeter in 3-5 days in $67.3 \%$ cases and in $14.9 \%$, it took $>7$ days for the platelet recovery. $77.5 \%$ patients had $a<20 \%$ rise in hematocrit during admission and $22.5 \%$ cases had $>20 \%$ rise. 


\section{JMSCR Vol||07||Issue||07||Page 511-518||July}

$22.6 \%$ had ascites, $25.7 \%$ had pleural effusion and $2 \%$ had pericardial effusion.

$16.1 \%$ had a past history of diabetes mellitus and were either on dietary modification or drugs.

Diabetes was detected after admission in $14.6 \%$ of cases. Total 105 cases were satisfying the ADA definition for diabetes among the cases.

$98 \%$ of the cases had an average blood sugar $<160 \mathrm{mg} \%$ during admission. $1.8 \%$ had a blood sugar $>360 \mathrm{mg} / \mathrm{dl}$ and $0.3 \%$ had sugars between 260-360mg/dl.

Among the diabetics, $0.6 \%$ had hypoglycemia, $1.5 \%$ had hyperglycemia and $0.6 \%$ had diabetic ketoacidosis.

97.1\% had an $\mathrm{HbA} 1 \mathrm{c}<7 \%$ and $2.5 \%$ had an HbA1c of $>7 \%$.

Figure 4: Final Diagnosis

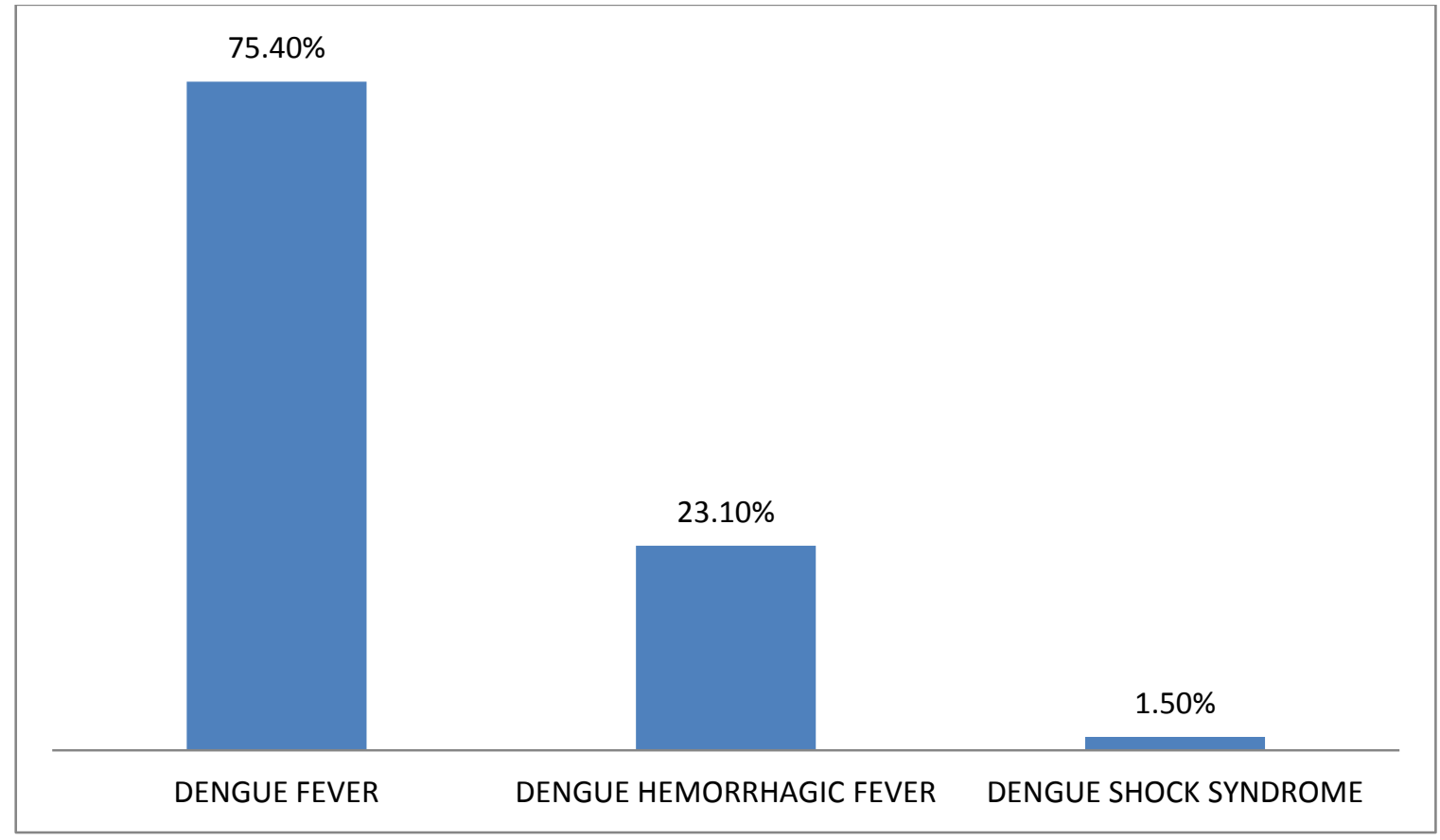

Figure 5: Final Outcome

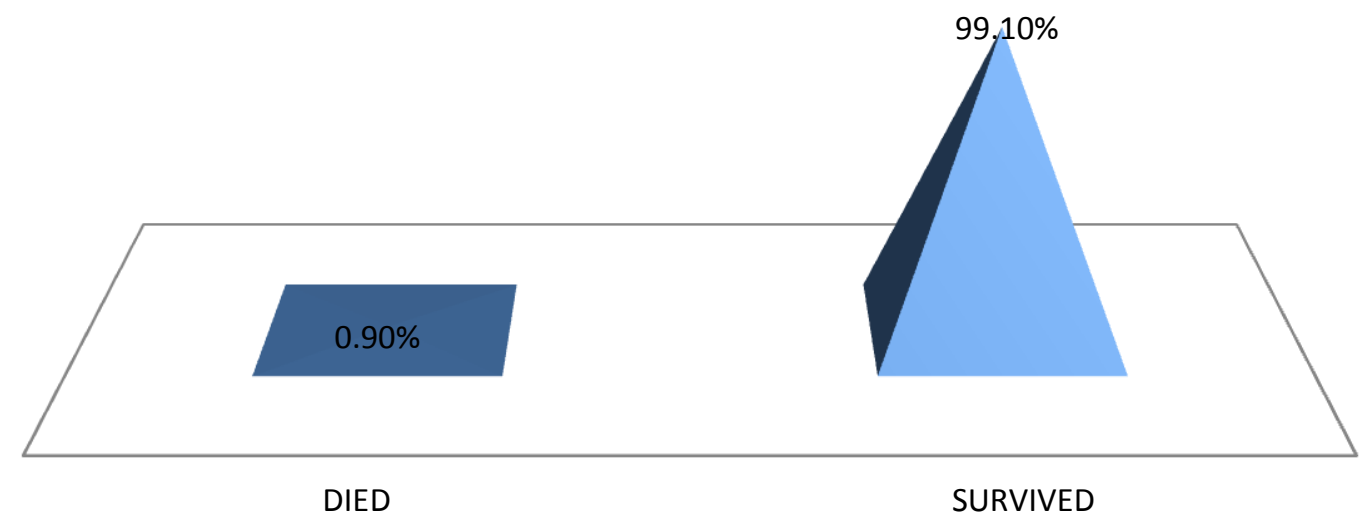




\section{JMSCR Vol||07||Issue||07||Page 511-518||July}

Table 1: Correlation between Diabetes and Outcome

\begin{tabular}{|l|c|c|c|c|}
\hline \multicolumn{2}{|c|}{ VARIABLE } & \multicolumn{2}{c|}{ OUTCOME } & \multirow{2}{*}{ P VALUE } \\
\cline { 3 - 4 } & & SURVIVED & DIED & \\
\hline \multirow{2}{*}{$\begin{array}{l}\text { DAIBETES } \\
\text { MELLITUS }\end{array}$} & PRESENT & 102 & 3 & \multirow{2}{*}{0.028} \\
\cline { 2 - 4 } & ABSENT & 237 & 0 & \\
\hline
\end{tabular}

Table 2: Relation between Diabetes and time Taken for Platelet Recovery

\begin{tabular}{|c|c|c|c|c|}
\hline \multicolumn{2}{|l|}{ VARIABLE } & \multicolumn{2}{|c|}{$\begin{array}{c}\text { TIME TAKEN FOR RECOVERY OF } \\
\text { PLATELET TO >70000/MM3 }\end{array}$} & \multirow{2}{*}{ P VALUE } \\
\cline { 3 - 4 } \multicolumn{2}{|c|}{} & $<7$ DAYS & $>7$ DAYS & \\
\hline \multirow{3}{*}{$\begin{array}{c}\text { DAIBETES } \\
\text { MELLITUS }\end{array}$} & PRESENT & 72 & 33 & \multirow{2}{*}{0.000} \\
\cline { 2 - 4 } & ABSENT & 219 & 18 & \\
\hline
\end{tabular}

Table 3: Relation between Diabetes and Rise in Hematocrit during Hospital Stay

\begin{tabular}{|c|c|c|c|c|}
\hline \multirow{2}{*}{\multicolumn{2}{|c|}{ VARIABLE }} & \multicolumn{2}{|c|}{ RISE IN HEMATOCRIT DURING HOSPITAL STAY } & \multirow[b]{2}{*}{ P VALUE } \\
\hline & & $<20 \%$ & $>20 \%$ & \\
\hline DAIBETES & PRESENT & 40 & 65 & \multirow[t]{2}{*}{0.000} \\
\hline MELLITUS & ABSENT & 225 & 12 & \\
\hline
\end{tabular}

Table 4: Relation between Diabetes and Presence of Pleural Effusion Clinically or Sonologically

\begin{tabular}{|c|c|c|c|c|}
\hline \multirow{2}{*}{\multicolumn{2}{|c|}{ VARIABLE }} & \multicolumn{2}{|c|}{ PLEURAL EFFUSION } & \multirow[b]{2}{*}{ P VALUE } \\
\hline & & YES & NO & \\
\hline \multirow{2}{*}{$\begin{array}{l}\text { DAIBETES } \\
\text { MELLITUS }\end{array}$} & PRESENT & 57 & 48 & \multirow{2}{*}{0.000} \\
\hline & ABSENT & 31 & 206 & \\
\hline
\end{tabular}

Table 5: Relation between Diabetes and Presence of Ascites Clinically or Radiologically

\begin{tabular}{|l|c|c|c|c|}
\hline \multicolumn{2}{|c|}{ VARIABLE } & \multicolumn{2}{c|}{ ASCITES } & \multirow{2}{*}{ P VALUE } \\
\cline { 3 - 4 } & YES & NO & \\
\hline \multirow{2}{*}{$\begin{array}{l}\text { DAIBETES } \\
\text { MELLITUS }\end{array}$} & PRESENT & 60 & 45 & \multirow{2}{*}{0.000} \\
\cline { 2 - 4 } & ABSENT & 31 & 206 & \\
\hline
\end{tabular}

Table 6: Relation between Diabetes Control and Pleural Effusion

\begin{tabular}{|c|c|c|c|c|}
\hline \multicolumn{2}{|c|}{ VARIABLE } & \multicolumn{2}{c|}{ PLEURAL EFFUSION } & \multirow{2}{*}{ P VALUE } \\
\cline { 3 - 4 } \multicolumn{2}{|c|}{} & YES & NO & \\
\hline \multirow{3}{*}{ HBA1C } & $<7 \%$ & 81 & 251 & \multirow{2}{*}{0.004} \\
\cline { 2 - 4 } & $>7 \%$ & 7 & 3 & \\
\hline
\end{tabular}

Table 7: Relation between Diabetes Control and Ascites

\begin{tabular}{|l|c|c|c|c|}
\hline \multicolumn{2}{|l|}{ VARIABLE } & \multicolumn{2}{|c|}{ ASCITES } & \multirow{2}{*}{ P VALUE } \\
\cline { 3 - 4 } & & YES & NO & \\
\hline \multirow{3}{*}{ HBA1C } & $<7 \%$ & 84 & 248 & \multirow{2}{*}{0.005} \\
\cline { 3 - 4 } & $>7 \%$ & 7 & 3 & \\
\hline
\end{tabular}


There was significant relation between the presence of diabetes (either newly detected or pre existing) between hemoconcentration, serositis, time for platelet recovery and outcome.

There was no significant association between diabetes and bleeding manifestations ( $p$ value 1.000)

The study could also not establish any significant relation between diabetes and the platelet count at the time of admission( $\mathrm{p}$ value 0.566 ) or the total WBC count at the time of admission( $p$ value $0.424)$. There was no significant relation between the glycemic control as expressed by the HbA1c and the presence of bleeding manifestations ( $p$ value 1.000). There was also no significant relation between the presence of diabetes or diabetic control with the development of pericardial effusion. The study could also not establish any significant relation between diabetic control and the final outcome ( $p$ value 1.000)

This study could not find any significant relation metformin use with bleeding manifestations, outcome or capillary leak.

\section{Discussion}

The study population included 342 cases, who satisfied the WHO case definition of Dengue.

In our study population, $62.3 \%$ were males and $37.7 \%$ were females. The disease affected mostly people belonging to the age group of 16-60 years. Most of the patients had fever duration ranging from 2- 7 days. The average stay duration in hospital was from 3-6 days.

Among the clinical features, $100 \%$ patients had fever. $91.5 \%$ patients had head ache and retro orbital pain. Musculoskeletal pain was seen in $83.6 \%$ cases.

Cough was an un common symptom seen in $8.2 \%$ cases. Bleeding manifestations were seen in $80 \%$ patients.

Seizure was seen in 2 cases. Of this one patient had a sub arachnoid hemorrhage. The second patient was a known seizure disorder who had a fever triggered seizure.
All the patients who got admitted had a platelet count of $<1$ lakh during admission. This is probably because ours is a referral centre. $50 \%$ of patients had total WBC count of $<4000 / \mathrm{mm} 3$.

$22.5 \%$ had a $>20 \%$ rise of hematocrit after admission. $67.3 \%$ cases had a recovery of platelet count to $>70000$ in 3-5 days time.

Evidence of capillary leak in the form of serositis were seen in $22 \%$ cases. Pleural effusion was the commonest form of serositis. Pericardial effusion was rare.

In our study group $16.1 \%$ were known Diabetics. 14.6\% satisfied the ADA definition of Diabetes following admission. Total number of diabetics were $30.7 \%$. $2.6 \%$ of the population had one or the other acute complications of diabetes like hypoglycemia, hyperglycemia or DKA.

Sub optimal blood sugar control as evidenced by an $\mathrm{HbA} 1 \mathrm{c}>7 \%$ were seen in $2.9 \%$ cases.

$75.4 \%$ cases were having Dengue Fever according to the WHO case definition. $23.1 \%$ had Dengue Hemorrhagic fever and $1.5 \%$ were having Dengue Shock Syndrome.

Of the 342 cases, 2 individuals with dengue shock syndrome expired. $99.1 \%$ cases survived and were successfully discharged.

It was seen that Diabetes, new onset or pre existing, was associated with an increased mortality, time taken for platelet recovery, capillary leak. However, no significant association was seen with the use of metformin and protective effect in dengue, as opposed to previous studies ${ }^{(10)}$ No significant association was observed between Diabetes and the total count or platelet count at the time of admission

\section{Conclusion}

Diabetes, pre existing or newly diagnosed, is significantly associated with mortality and complications, in patients admitted with Dengue.

\section{References}

1. World Health Organisation, Dengue guidelines for diagnosis, treatment, 
prevention and control. Geneva: World Health Organisation: 2009

2. Murray NE, Quam MB, Wilder- Smith A. Epidemiology of Dengue: Past, present and future prospects. Clin Epidemiol 2013; 5: 299-309.

3. World Health Organisation, Dengue hemorrhagic fever: diagnosis, treatment, prevention and control. $2^{\text {nd }}$ ed. Geneva: world health organization 1997.

4. Lee IK, Liu JW, Yang KD. Fatal dengue hemorrhagic fever in adults: emphasizing the evolutionary pre fatal clinical and laboratory manifestations. PLoS Neglected Trop Dis 2012: 6; 1532

5. Tsai Cy, Lee IK, Lee CH, Yang KD, Liu JW. Comparisons of dengue illness classified based on the 1997 and 2009 World Health Organisation dengue classification schemes. J Microbiol Immunol Infect 2013; 46:271e 81.

6. Figueiredo MA, Rodrigues LC, Barreto ML, Lima JW, Costa MC, MOrato V et al. Allergies and diabetes as risk factors for Dengue Hemorrhagic fever: Results of a cse control study. PLoS Neglected Tropical Disease. 2010;4: e699

7. Pang J, Salim A, Lee VJ, Hibberd ML, Chia KS, Leo YS et al. diabetes with hypertension as a risk factor for adult dengue hemorrhagic fever in a predominantly serotype 2 epimdemic: A case contro; study: PLoS Neglected Tropical Disease. 2012;6: e 1641

8. Limonta D, Torres G, Capo V, Guzma n MG. apoptosis, vascular leakage and increased risk of severe dengue in a type 2 diabetes mellitus patient. Diabetes Vasc Dis Res 2008;5: 2 13e 4
9. Htun NS, Odermatt P, Eze IC et al. Is diabetes a risk factor for a severe clinical presentation of dengue? Review and mta analysis. PLoS Neglected tropical disease. 2015;9:e0003741

10. Shih CJ et al. Association between the use of oral anti diabetic agents and the risk of sepsis: A nested case-control study. Scientific reports 2015:5;15260. 